\title{
Traumatic thrombosis of the internal carotid artery in the neck
}

\author{
J. TREVOR HUGHES AND BETTY BROWNELL
}

From the Department of Neuropathology, Radcliffe Infirmary, Oxford

Hugh Cairns (1942), in describing the vascular aspects of head injuries, referred to damage to the internal carotid artery, which might be injured either in the neck or within the skull in connexion with fractures of the base. Trauma to the internal carotid artery in the neck can produce thrombosis or embolism, and this may result in ischaemia or infarction affecting the corresponding cerebral hemisphere. Cases of this nature may be caused by a direct penetrating wound involving the artery in the neck, but also may be associated with an injury which has not breached the skin of the neck. The clinical importance of this syndrome is that the effects of the carotid artery obstruction may be falsely attributed to direct injury to the brain or spinal cord. We present here three cases of this type in which the nature of the injury and the subsequent cerebral infarction were studied clinically and at necropsy. In case 1 there was a penetrating neck wound, while in cases 2 and 3 the carotid artery had been injured without a skin laceration.

CASE 1

This pensioner (E.F., A. 1728), at the age of 21 in September 1914, enlisted in the British Army and joined the Rifle Brigade. On 3 June 1916 he was wounded at Ypres in Belgium, receiving a wound in the left side of the neck from a bullet which caused a left facial palsy, deafness in the left ear, and paralysis of the right arm and leg. The bullet was removed at a field hospital in France, and he was evacuated to England on 15 June 1916 , by which time the left-sided deafness and facial weakness had recovered. He had also regained the movement of the right leg, but the right arm remained completely paralysed and anaesthetic. The neck wound healed and he was discharged from the Army on 16 October 1916. He was awarded a disability pension in 1918, when he was found to have a 3 in. scar on the left side of the neck, slight weakness of the left side of the face, and weakness of the left sternomastoid and trapezius. There was wasting of the muscles of the right arm with some loss of co-ordination. He was considered at this time to have had a contusion affecting the left side of the medulla and the spinal cord. A radiograph showed no evidence of a bony injury or of a foreign body in the region of the old wound in the left side of the neck. Subsequently he obtained employment and he remained at work until 1945. In December 1951 he was found to have diabetes mellitus of a mild type which was controlled with regular insulin therapy. He was then unable to work because of general disability and the paralysis of his right hand, some weakness of the right leg having also appeared at this time. For some time he had complained of angina, and this further limited his exertion capacity. On 5 March 1953, a medical report indicated that he had an upper motor neurone weakness of both the right arm and the right leg, with an increase in tendon reflexes on the right side, and a right extensor plantar response. There was also some impairment of position sense on the right side. He had some arthritis of his left shoulder causing him pain, and electrocardiographic evidence of an anterior myocardial infarction. His blood pressure was $130 / 100$. His final medical examination by the Ministry of Pensions took place on 24 August 1965 when he complained of forgetfulness, lack of concentration, irritability, and occasional headaches. His limb paresis now prevented him from walking more than a few yards. His neurological state was unchanged, except that he had slight bilateral deafness of the conduction type. His last admission was to a hospital in Carshalton where he died on 25 November 1965. A necropsy was performed and the fixed brain was sent to us by the Department of Pathology, St. Helier Group of Hospitals, Carshalton, Surrey.

\section{NECROPSY FINDINGS}

Fixed Brain The external examination showed an old softening in the left cerebral hemisphere and a recent haemorrhagic infarct on the right side. At the base of the brain, the vessels of the circle of Willis were of normal pattern, but showed moderately severe atheroma, particularly affecting the internal carotid arteries.

The brain in coronal slices showed two vascular lesions, one in each hemisphere (Fig. 1). On the left side, there was a very old vascular softening, involving most of the territory of the left middle cerebral artery. What remained was a cavity in the central part of the hemisphere, communicating with the body of the lateral ventricle, and lined by a mixture of ependyma, meninges, and some fibrous tissue. Branches of the left middle cerebral artery could be seen cut across, but no occluded vessels were 


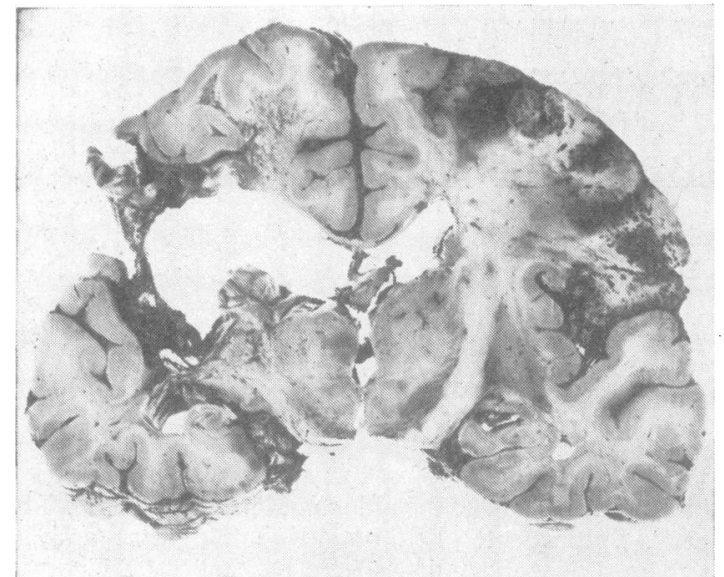

FIG. 1. Case 1. Posterior aspect of a coronal slice of the cerebrum at the level of the third ventricle. On the left side there is a cavity from an old vascular softening in the territory of the middle cerebral artery. There is acute infarction on the right side. $\times 0.4$.

visible. This old vascular lesion probably dated from the injury in the first world war, but was clearly an old infarction and there was no evidence of a cerebral traumatic lesion. The thalamus on the left side was considerably smaller than that on the right, due to secondary degeneration. The corpus callosum had also suffered atrophic degeneration and was very thin. The lesion in the right hemisphere was a recent haemorrhagic infarct, involving the territory supplied by the distal branches of the right middle cerebral artery. The only abnormality in the brain-stem was that the left pyramid was shrunken and grey. The cerebellum was normal.

In histological sections, the old infarct on the left side was seen as a large astrocytic glial scar, involving cortex and white matter by total destruction of neurone cell bodies, axons, and myelin tubes (Fig. 2). On the right side there was recent haemorrhagic infarction involving the cerebral cortex and white matter. Both these infarcts were in the territories of the middle cerebral arteries, but no occlusions were visible in the branches of these vessels. Sections of the midbrain, pons, and medulla showed Wallerian degeneration in the left pyramidal tract.

\section{CASE 2}

This 40-year-old motor cyclist (A.K., N.8508) was admitted to hospital 45 minutes after a road accident. He was alert and orientated, and showed no abnormal neurological signs. There were cuts over the left side of the face, a fracture of the right malar bone, and tenderness over the angles of the jaw, and over the sternum. Twelve hours after the accident he was found to have a hemiplegia which had probably developed during sleep. On examination, he was drowsy and had a left hemiplegia and hemianaesthesia, complete in the face and arm,

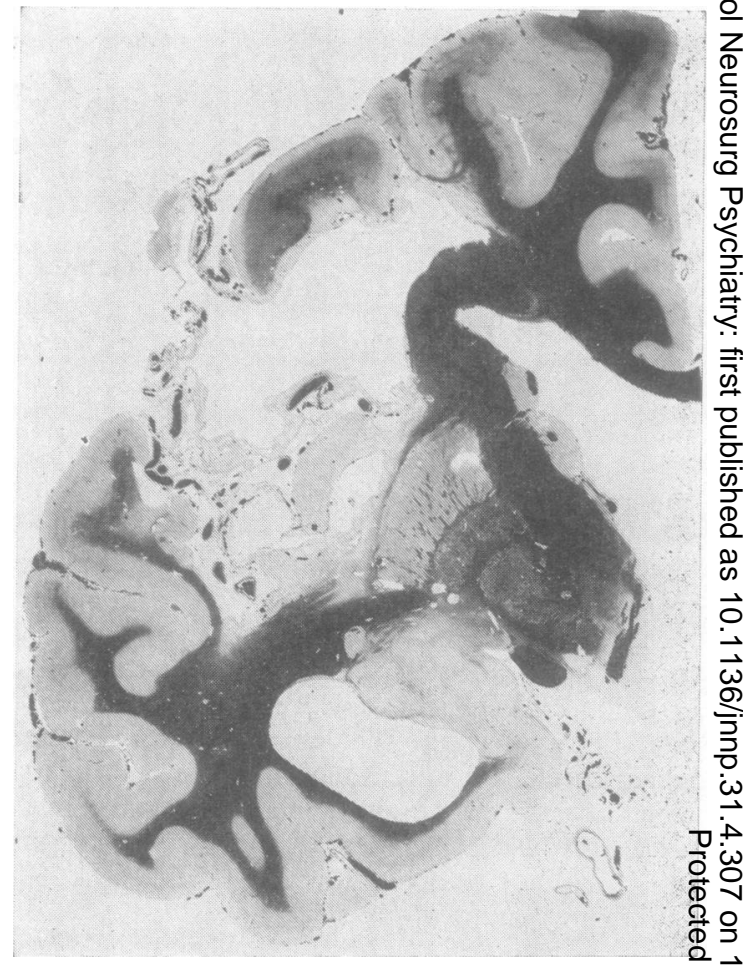

FIG. 2. Celloidin-embedded section, stained for myelin, left cerebral hemisphere of case 1. Kultschitzky-Pal, $\times 1 . \otimes$

but incomplete in the lower limb. There was also a le homonymous hemianopia with defective conjugate movement of the eyes to the left. His blood pressure was $115 / 70$, his pulse 66 , and his retinal artery pressure on the left was 90/50 whereas on the right it was 20/10. Carotid pulsation appeared equal on both sides. The diagnosis of right carotid thrombosis was suspected from $\frac{\circ}{\Phi}$ the time of his readmission, because of the profound $\varrho$ hemisphere signs and the relative preservation of his $\overrightarrow{\vec{O}}$ conscious level. The retinal artery pressure readings $\exists$ supported this diagnosis, which was confirmed by $\supsetneq$ bilateral carotid angiography. On the right side there was a complete obstruction of the internal carotid artery halfway along its course, and the left cerebral $\overline{3}$ arteriogram showed a minimal displacement of the ? midline vessels to the left with only a very slight amount of filling of the right anterior cerebral artery. There was a little filling of the branches of the right pericallosal artery. Twenty-four hours after the accident the right $₹$ carotid arteries were explored. A small opening was made 0 at the bifurcation and there was virtually no back bleeding from the right internal carotid artery, and $\frac{D}{O}$ pulsation could be felt for only $1 \frac{1}{2}$ in. upwards. A corkscrew reamer was then passed up the internal carotid artery and $N$ the clot was removed, after which there was a sudden powerful jet of back bleeding. The operation was done $\tilde{O}$ under heparin therapy and this was continued for a 
further 24 hours while oral Dindevan was started. Four hours after the operation he had recovered from the anaesthetic and was again alert and fully orientated, but with unchanged neurological signs. His general condition was maintained up to 17 hours after the operation when he became drowsy, restless, and unable to speak more than a few words. His respiration appeared obstructed and a tracheostomy was performed, but without improvement. He continued to deteriorate and 48 hours after his accident his right pupil became fixed and dilated. In spite of treatment with intravenous urea and cooling to $90^{\circ} \mathrm{F}$ he became decerebrate and remained so, developing a profound metabolic disturbance which ended in his death six days after the accident.

NECROPSY FINDINGS In addition to the lacerations and fractures described in the clinical report, a transverse fracture across the middle of the sternum was found, with underlying haemorrhage into the anterior mediastinum. The skull was intact apart from the fracture of the right malar bone. Other abnormalities were confined to the cardiovascular and nervous systems. The heart appeared normal. The aorta and major arteries were mildly affected by atheroma in the form of small plaques without ulceration or calcification. Linear deposits of this nature were present in both common carotid arteries, but not in their internal or external branches. Both common carotid arteries had been entered by angiogram needles which had not caused any untoward local effect, and the left internal carotid artery contained merely post-mortem thrombus.

Right internal carotid artery The right internal carotid artery was embedded in an operative haematoma, $4 \mathrm{~cm}$ in diameter and $10 \mathrm{~cm}$ in length, which had spread between the muscles of the neck and around the right lobe of the thyroid. A sutured incision extended for $1.5 \mathrm{~cm}$ upwards from the carotid bifurcation. Situated $2.5 \mathrm{~cm}$ above the bifurcation, on the anterior wall of the vessel, was a transverse intimal tear $0.4 \times 0.4 \mathrm{~cm}$ in area, with attached blood clot extending into the vessel wall (Fig. 3). Apart from this clot, there was no thrombus remaining in the lumen of the vessel. Histological sections of the artery in the region of the intimal tear showed that this extended into the tunica media, where there was a haematoma of moderate size (Fig. 4).

Fixed brain The brain weighed $1,800 \mathrm{~g}$ and there was swelling of the right hemisphere causing subtentorial herniation of the right uncus. The vessels of the circle of Willis were normal. Coronal slices of the brain showed recent softening in the territory of the right middle cerebral artery, with sparing of the anterior and posterior cerebral territories on that side, and no signs of infarction in the left hemisphere (Fig. 5). The infarction was most intense in the centre of the cerebral hemisphere, and this was attributed to the complete blockage of the right lateral striate arteries at their origin. The brain-stem showed considerable lateral compression, with midline bruising, due to herniation of the right uncus. A histological section of the region of the right insula showed changes of acute infarction, and in the same section, a branch of the middle cerebral artery was seen to be partially blocked by organizing blood clot. This clot

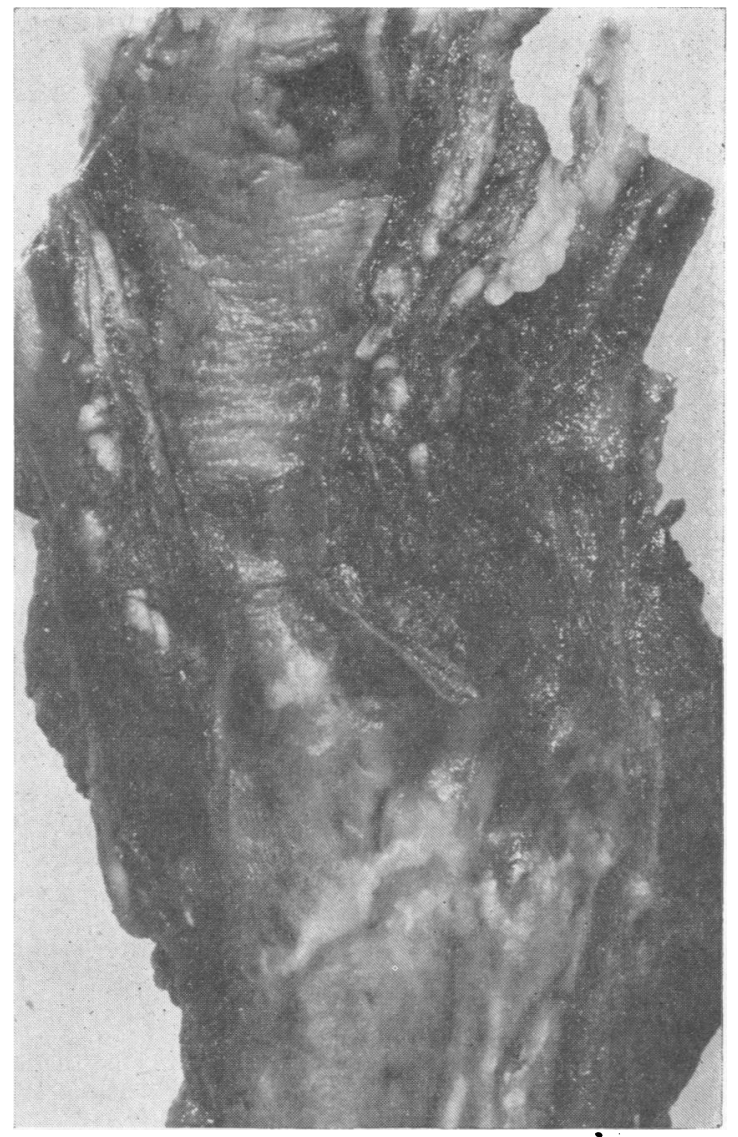

FIG. 3. Case 2. Right carotid arteries opened and viewed from the posterior aspect. There is an intimal tear in the internal carotid artery $2.5 \mathrm{~cm}$ above the bifurcation. $\times 3$.

was irregular in shape and was not attached to the vessel wall, and for these reasons it was considered to be an embolus.

\section{CASE 3}

A 29-year-old man (D.R., N. 8981) was admitted to hospital 30 minutes after a car accident. He was fully conscious and orientated, with no abnormal neurological signs, and complained only of pain in the chest on breathing. There was a small cut at the angle of the left mandible, but radiographs revealed no fracture of jaw or skull. Thirty-two hours after the injury he was found to have a right hemiplegia and hemianaesthesia, right homonymous hemianopia, and an expressive aphasia. A left carotid angiogram, performed 37 hours after the accident, showed a marked irregular narrowing of the internal carotid artery in the neck with no contrast medium reaching the cerebral circulation. The right carotid angiogram showed a moderate shift of the 


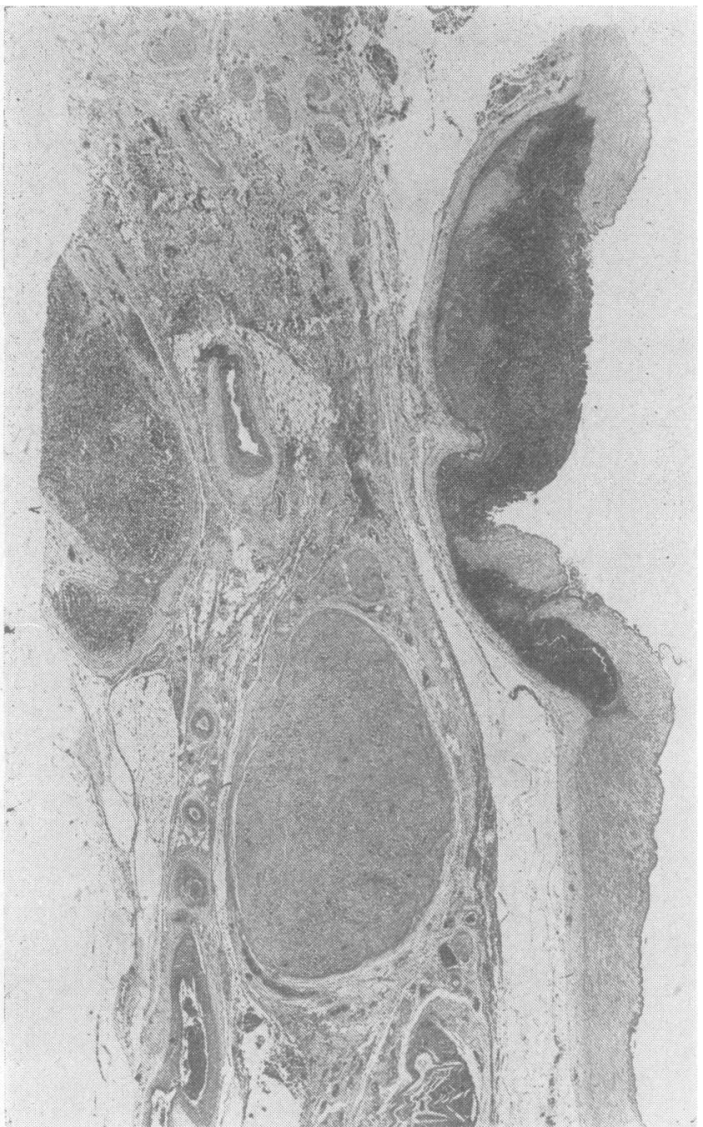

FIG. 4. Case 2. Longitudinal section of the internal carotid artery through the intimal tear. There is a large haematoma in the tunica media beneath the intimal breach. Van Gieson and elastic, $\times 10$.

midline vessels to the right with very little spontaneous cross circulation. He held his own for another five hours but then deteriorated, becoming decerebrate 42 hours after his accident. In spite of a tracheostomy, intravenous urea, and cooling to $90^{\circ} \mathrm{F}$, his pupils became fixed and dilated and he died 72 hours after the accident.

NECROPSY FINDINGS A necropsy was carried out 48 hours after death. Lacerations and bruising were seen as described in the clinical report, and fractures were present through the right third and fourth costochondral junctions, and transversely through the middle of the sternum. There was haemorrhage into the anterior mediastinum, with retropharyngeal haemorrhage and oedema of the pharynx and glottis. There was contusion of the right lung near the hilum, and both lungs showed basal collapse and scattered subpleural haemorrhages. The heart, aorta, and major arteries, with the exception of the internal carotid arteries, were normal, with negligible atheroma.

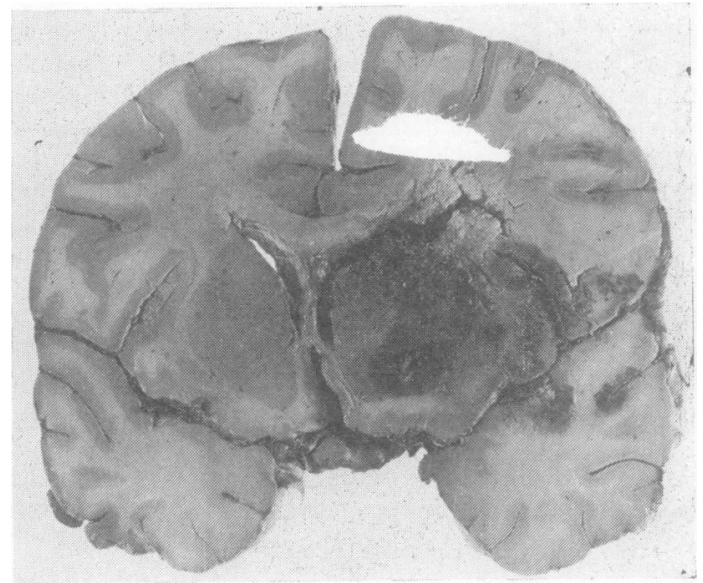

FIG. 5. Case 2. Posterior aspect of a coronal slice of the cerebrum at the level of the optic chiasm. There is recent softening in the whole territory of the right middle cerebral artery. Half life size.

Left internal carotid artery The left internal carotid artery contained grey thrombus commencing at a poin $4 \mathrm{~cm}$ above the origin of the vessel, and extending upwards for about $2 \mathrm{~cm}$. Sections through the lower en of the thrombus showed fresh haemorrhage surrounding the artery, intimal damage with a polymorph reaction and haemorrhage in the tunica media which encircle $\$$ half of the lumen. The thrombus showed earl organization.

Fixed brain The brain weighed $1,460 \mathrm{~g}$, and was swollen, with tentorial grooving of both unci and prominence of the cerebellar tonsils. The circle of Willis was abnormal in that the left posterior cerebral artery was mainly fed from the internal carotid artery through a large posterior communicating artery. The terminal portion of the left internal carotid artery contained grey ante-mortem thrombus which, from its position, was considered to be an embolus lodging at the bifurcation of the artery.

Coronal slices showed the cause of the cerebral swelling to be recent infarction of the whole of the territories of the left middle and posterior cerebral arteries (Fig. 6). The anterior cerebral territory was spared, this region being fed from the right side via the anterior com-? municating artery. In the centre of the left hemisphere was a region of haemorrhagic extravasation situated in the territory of the left lateral striate arteries, which were directly obstructed by the embolus at their origin. The brain-stem showed lateral compression due to the $\bigcirc$ subtentorial herniation. In histological sections of the left cerebral hemisphere, changes of infarction were $\frac{D}{O}$ seen in the middle and posterior cerebral territories, with more severe infarction centrally in the region $N$ supplied by the lateral striate arteries. Ante-mortem thrombus (Fig. 7) was found in a main branch of the left middle cerebral artery in the Sylvian fissure. 


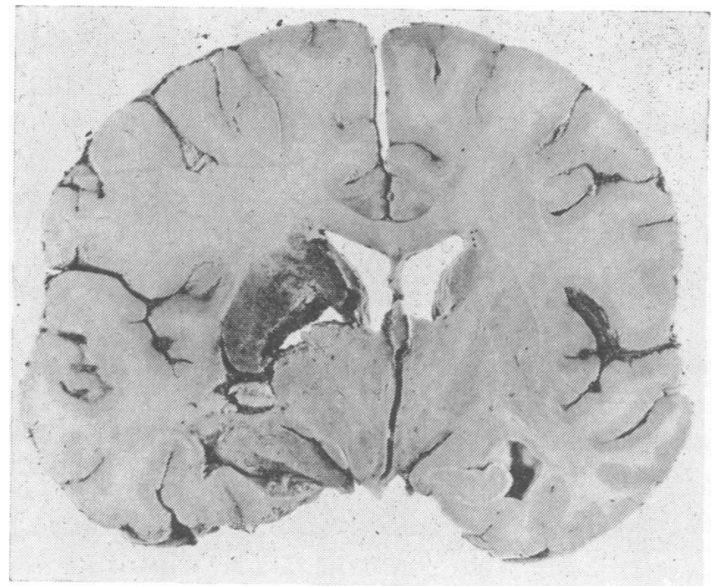

FIG. 6. Case 3. Posterior aspect of a coronal slice of the cerebrum at the level of the third ventricle. There is recent softening, seen as pallor, in the territory of the left middle cerebral artery. As in Fig. 5, the territory of the lateral striate arteries is outlined by intense haemorrhagic infarction. Half life size.

\section{REVIEW OF THE CLINICAL AND PATHOLOGICAL FINDINGS}

THE NEUROLOGICAL SYNDROME In all three cases described in this report, an injury to one internal carotid artery in the neck had given rise to a neurological syndrome caused by infarction in the appropriate cerebral hemisphere. This neurological syndrome was a dense paralysis and sensory loss in one side of the body which was accompanied by hemianopia in Cases 2 and 3, and by aphasia in Case 3. The time of onset of the neurological deficit was not known accurately in case 1 (battle casualty), but it was apparent at his first full medical examination on the battlefield. In Cases 2 and 3 the onset of the hemisphere disturbance occurred 12 and 32 hours respectively after a road accident, and in both cases had apparently developed during sleep. Case 1 recovered from the effects of his war injury and lived for a further 52 years, during which period he suffered from slight weakness of the right hand and leg. Cases 2 and 3 died six days and 72 hours respectively after their accidents without any improvement in their neurological state. In both these cases death was due to the brain swelling caused by the cerebral infarction.

NATURE OF THE INJURY In Case 1 it is evident from the records of a British Army surgeon that there was an extensive penetrating wound on the left side of the neck, from which a bullet was extracted.

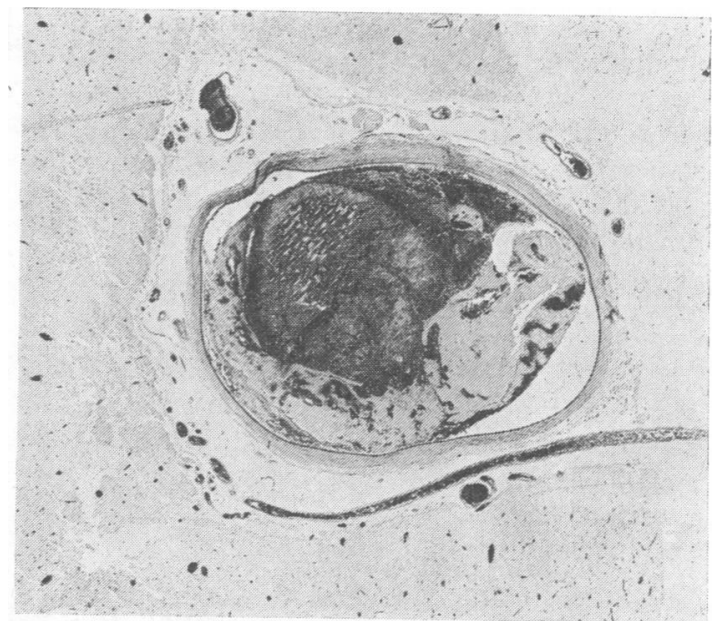

FIG. 7. Photomicrograph of transverse section of left middle cerebral artery in the Sylvian fissure. The lumen is blocked by embolic thrombus. Haematoxylin and eosin, $\times 14$.

The wound had caused a left facial nerve lesion and deafness on the left side, in addition to the syndrome of cerebral infarction under discussion here. In Cases 2 and 3 there was no penetrating wound in the neck and the internal carotid artery had apparently been injured indirectly by the trauma. There was no external bruising visible in the neck and the nature of the injuries suggested a different mechanism of trauma. We were impressed by the coincidence in both cases of lacerations and bruising around the chin and mandible with a fracture of the sternum. These findings suggested that during the accident there had been a movement in which the carotid vessels on one side were pulled downwards, while the head was thrown into hyperextension or extreme lateral flexion.

PATHOLOGICAL FINDINGS IN THE CAROTID ARTERIES The neck arteries of Case 1 were not explored at necropsy. The findings at necropsy in Cases 2 and 3 were remarkably similar in most respects. The internal carotid artery was embedded in haematoma which in Case 2 was partly a sequel of the previous surgical operation of thrombarterectomy. In both cases there was a haematoma in the tunica media communicating through an intimal tear with the lumen of the vessel. Ante-mortem thrombus was present in the internal carotid artery of Case 3. Similar thrombus had been removed at operation from Case 2.

PATHOLOGICAL FINDINGS IN THE BRAIN All three cases showed cerebral infarction in the distribution 
of a middle cerebral artery and this, in Case 1, took the form of an old healed cavity. In Cases 2 and 3 the arterial obstruction was due to the lodgement of an embolus in the terminal part of the internal carotid and the origin in the middle cerebral artery, and this had produced the picture of softening in the middle cerebral territory with intense infarction in the territory of the lateral striate arteries.

\section{REVIEW OF LITERATURE}

Thrombosis of the carotid artery due to a penetrating wound of the neck is a complication which is seen most frequently when large numbers of battle casualties are dealt with. In the first world war, Makins (1919) was familiar with this type of case and Caldwell and Hadden (1948) saw eight similar cases in the second world war. The latter, who were treating casualties in Europe at the 5th Evacuation Hospital, found these eight cases among over 25,000 battle casualties treated during a period of 15 months. Of these eight cases, five were confirmed by necropsy examination of the carotid arteries, which contained ante-mortem thrombus. In several cases, the thrombosis extended throughout the carotid artery as far as its termination. The local damage to the carotid artery in the neck was usually extensive and intimal tears were found which sometimes showed separation of the intima and curling up of the free edge into the more distal part of the damaged artery. Ecker (1945), in reporting his experience with second world war casualties, makes the point that penetrating wounds in which a high-velocity missile does not actually strike the internal carotid artery may yet induce thrombosis from the indirect trauma of the missile passing the artery. These cases of the type described by Ecker (1945) form a connecting link with the second group that we shall now review.

This group, in which the carotid artery is injured indirectly, and where there is no penetrating wound in the neck, is less well recognized. The first case of this nature in the literature is probably that of Verneuil (1872). In this case, after a railway accident with multiple injuries, left cerebral infarction was caused by thrombosis of the left internal carotid artery. There was no laceration or bruising of the neck but the outline of the left sternomastoid muscle was obscured by swelling. At necropsy, thrombus was found in the left internal carotid artery $2 \mathrm{~cm}$ distal to the carotid bifurcation. The external wall of the vessel was intact but the intima and the media were torn. Verneuil suggested that a sudden wrenching of the neck might have caused this tear. A similar case was reported by Greco (1935) whose patient, a 23-year-old butcher, was knocked from his bicycle, receiving cuts to his chin and lower lip 으 with tenderness of his left mandible. He remounted $Z_{\mathbb{D}}$

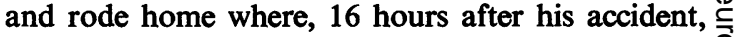
he developed a right hemiplegia which led to his of death. Necropsy showed a transverse laceration of the tunica intima and media of the left internal 0 carotid artery, which contained ante-mortem thrombus. Subsequently, further cases in which $\stackrel{\odot}{\leq}$ necropsy was performed have been added to these two early cases and the literature was reviewed by? Murray (1957), who added one case to a review of $\overrightarrow{\vec{F}}$ eight cases with recorded necropsy findings. Murray $\stackrel{\text { ? }}{+}$ did not mention a case by Egas Moniz (1941), and subsequently a further case was added by Toakley $\frac{\bar{\sigma}}{\bar{\omega}}$ and McCaffrey (1965).

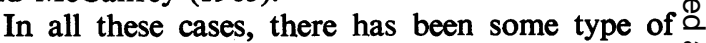
violent accident involving the head or the neck and $\%$ sometimes the clavicle or upper thoracic cage. There $\vec{\circ}$ has usually been an interval of several hours before the development of the neurological syndrome. $\vec{\omega}$ In the more recent reports angiography, when performed, has shown a blocked internal carotid artery. The necropsy findings have been, in the majority of reports, very similar. The affected cerebral hemisphere has contained a very large $\vec{\omega}$ infarct in the appropriate arterial territory. Tlg carotid artery concerned has usually shown 90 tear affecting the tunica intima and tunica medi but not involving the adventitial coat of the arters. Ante-mortem thrombus has always been demor-co strated within the injured vessel, and has usual of been confined to the injured internal carotid arter俤

\section{COMMENT}

Three points of interest merit further discussion.

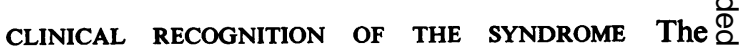
clinical features of this syndrome are important in $\overrightarrow{\overrightarrow{0}}$ distinguishing it from the more common case of $\frac{0}{3}$ head injury with direct cerebral damage. As we have $?$ mentioned earlier, the external injuries have been remarkably similar in many of the cases. It has also been possible to recognize the syndrome from the spectrum of the neurological signs and their mode of onset. The diagnostic features are the rapid 3 . onset of a hemiplegia with the relative preservation of the level of consciousness, and the absence, at least initially, of signs of cerebral compression. Confirming signs are the absence of carotid pulsation and the findings on ophthalmodynamometry. Bilateral angiography will demonstrate the original intravascular thrombosis in the internal carotid $N$ artery in the neck. This procedure may also help in N showing a subsequent embolus in the middle cerebral $\mathrm{\omega}$ 
artery and the amount of cross-filling from the other side.

MECHANISM OF INJURY In cases such as Case 1, in which there is a direct penetrating wound injuring the carotid artery, the mechanism of the injury is relatively simple, and no elaborate theories are required to explain why there is thrombosis in the injured artery. The sequel of thrombosis following trauma is similar to that seen in other arteries. In this type of injury the trauma of the close passage of a high velocity missile may cause thrombosis in a vessel which is not directly struck.

Cases 2 and 3 belong to the second group, in which the carotid artery is injured indirectly without a penetrating wound in the neck. In both cases there are clues in the clinical and the pathological examinations to the mechanism of the injury. There was evidence of trauma to the chin and angle of the jaw, and of injury to the sternum, which was found to be transversely fractured in both cases. These cases may be compared with others in which fractures of the thoracic cage were found. If one visualizes an injury in which there is a blow to the angle of the jaw with the head being thrown backwards, and a simultaneous injury to theanterior thorax with a fracture of the sternum, it seems clear that there is a mechanism involved in which the carotid arteries on one side could be forcibly stretched. The necropsy findings in the affected carotid artery were consistent with a stretching injury, since in both these cases there was a tear of the intima and media, but no damage to the adventitia. We have discussed in the review of the literature how frequently such findings have been noted in cases of indirect trauma to the carotid artery. We have conducted experiments on cadavers and these show that when the head is thrust forcibly backwards and to one side, the carotid arteries on the opposite side are stretched. The effect is magnified if the sternum is sawn through transversely-simulating fracture -when pressure on the lower part of the sternum tethers the innominate artery.

The way in which this tear of the tunica intima and media will cause thrombosis requires some consideration. It is not certain whether an intimal tear is necessarily important, and Poole, Sanders, and Florey (1958) in their experiments to remove the tunica intima of the aorta of rabbits, found that if damage to the other coats of the vessel was avoided thrombosis rarely occurred. Honour and Mitchell (1963) found that damage to the tunica media was the more important feature, and suggested that a chemical stimulus was exerted by some component of damaged smooth muscle. It may be that this is so, and that the intimal tear is of some importance in allowing this substance easy access to the arterial lumen where it will cause the formation of a thrombus.

THE CAUSE OF THE CEREBRAL INFARCTION Some explanation is required as to why the obstruction of one internal carotid artery results in cerebral infarction. Experience relevant to this problem has been gained from the treatment, by surgical ligation of a carotid artery, of subarachnoid haemorrhage due to intracranial aneurysm. It is relatively uncommon for patients with healthy arteries to develop a cerebral infarction following ligation of the internal carotid artery in the neck, provided there is an adequate anastomotic circulation around the circle of Willis. In these circumstances the cerebral hemisphere on the side of the ligation will usually receive an adequate blood supply from the other internal carotid artery or the vertebral arteries.

In both Cases 2 and 3 the evidence at necropsy showed that the most significant obstruction to the cerebral circulation was situated in the middle cerebral artery-distal to the anastomosis of the circle of Willis-and was caused by an embolus (Fig. 8). Obstruction at this particular point will almost always cause infarction of the corresponding middle cerebral artery territory. The additional feature seen in cases of embolus at this particular point is severe haemorrhagic infarction in the territory of the lateral striate arteries, which arise from the middle cerebral artery at this point. A

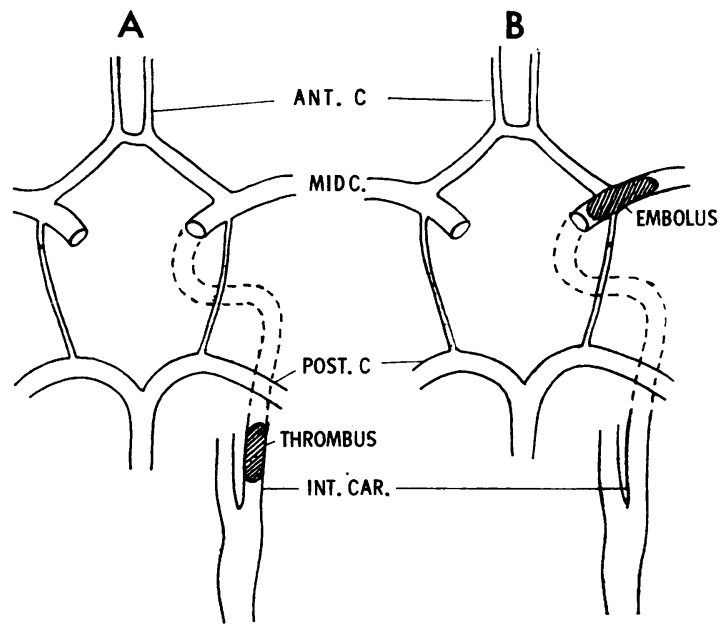

FIG. 8. Diagram of major arteries supplying the brain. In Fig. 8A, the proximal obstruction of the internal carotid artery does not usually cause cerebral infarction, because of the anastomosis provided by the circle of Willis. In Fig. 8B, the obstruction is more distal and there will be infarction in the territory of the middle cerebral artery. 
feature of both cases 2 and 3 was that, superimposed on the rather pale infarction in the middle cerebral territory, was an intense haemorrhagic infarction of the central territory of the lateral striate arteries.

\section{SUMMARY}

Three cases of cerebral infarction due to traumatic internal carotid thrombosis in the neck are described. In the first case, the mechanism of the injury was a direct penetrating wound, while in the subsequent two cases, indirect trauma was responsible. The mechanism of the injury to the internal carotid artery in these two last-mentioned cases is discussed, and evidence is presented that the cerebral infarction occurred as a result of embolism from the proximal into the distal carotid circulation. The recognition of this clinical syndrome is discussed.

Dr. Brownell is receiving a grant from the National Fund for Research into Poliomyelitis and other Crippling Diseases.

The authors thank the Department of Pathology, St. Helier Group of Hospitals, Carshalton for Case 1, and the consultants of the United Oxford Hospitals for the use of their clinical records of Cases 2 and 3.

\section{REFERENCES}

Cairns, H. (1942). The vascular aspects of head injuries. Lisboa med., $19,375-410$.

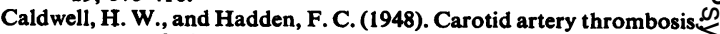
report of eight cases due to trauma. Ann. inter. Med., 28@ 1132-1142.

Ecker, A. D. (1945). Spasm of the internal carotid artery. J. Neurosurg. 2, 479-484.

Egas Moniz, A. (1941). Trombosis y Otras Obstrucciones de las: Carotidas. Salvat Editores, Barcelona.

Greco, T. (1935). Le trombosi post-traumatiche della carotide. Arch $\stackrel{\text { S }}{\circ}$ ital. Chir., 39, 757-784.

Honour, A. J., and Mitchell, J. R. A. (1963). Platelet clumping in

vivo. Nature (Lond.), 197, 1019-1020.
Makins, G. H. (1919). On Gunshot Injuries to the Blood Vessels. $\frac{\widehat{\Phi}}{\bar{D}}$ John Wright, Bristol.

Murray, D. S. (1957). Post-traumatic thrombosis of the internal carotid and vertebral arteries after non-penetrating injuries of the neck. Brit. J. Surg., 44, 556-561.

Poole, J. C. F., Sanders, A. G., and Florey, H. W. (1958). Thee regeneration of aortic endothelium. J. Path. Bact., 75, 133-143.-

Toakley, G., and McCaffrey, J. (1965). Traumatic thrombosis of the internal carotid artery. Aust. N.Z. J. Surg., 34, 261-264.

Verneuil, A. A. S. (1872). Contusions multiples, delire violent, hémiplégie à droite, signes de compression cérébrale. Mort le cinquième jour. Rupture complète des tuniques profondesర de la carotide interne gauche au cou. Oblitération des vaisseaux $\omega$ au point lésé par un caillot qui remonte jusqu'aux dernières.branches de l'artère sylvienne: ramollissement cérébragit étendu à la presque totalité du lobe moyen, Bull. Acad. Med. W (Paris) (2nd ser.), 1, 46-56. 